Review article

\title{
SIRS, CARS and MARS in relationship to cancer cachexia and its clinical implications
}

\author{
Masahiko Shibata $^{1,2)}$, Kenji Gonda ${ }^{2)}$, Tatsuo Shimura ${ }^{3,4)}$, Kenichi Sakurai ${ }^{5)}$, and Seiichi Takenoshita ${ }^{6}$ \\ ${ }^{1)}$ Departments of Advanced Cancer Immunotherapy, ${ }^{2)}$ Gastrointestinal Tract Surgery, ${ }^{3)}$ Progressive DOHaD Research, \\ and ${ }^{4)}$ Hepato-Biliary-Pancreatic and Transplant Surgery, Fukushima Medical University \\ ${ }^{5)}$ Division of Breast and Endocrine Surgery, Nihon University School of Medicine \\ ${ }^{6)}$ Fukushima Medical University
}

\begin{abstract}
Cachexia is a syndrome characterized by continuous, involuntary weight loss and systemic inflammation. Several mediators that are either host- or tumor-derived, such as pro-inflammatory cytokines, have been implicated in the pathogenesis of cancer cachexia.

The compensatory humoral system that typically reduces the excessive production of pro-inflammatory cytokines in order to maintain homeostasis is also highly activated in patients with cancer cachexia. The above observations, along with cachexia, suggest that conditions similar to SIRS (systemic inflammatory response syndrome) and CARS (compensatory anti-inflammatory response syndrome), or to mixed anti-inflammatory response syndrome (MARS), may exist in cachexia. The typical immunological status of patients with cancer cachexia has been demonstrated to be suppressed cell-mediated immunity, and a Th2-dominant condition and myeloid-derived suppressor cells: MDSCs are major causes of this suppression.

Several therapies and strategies have been proposed with regard to anti-inflammatory treatments. However, no effective therapy against cancer cachexia is currently available. Further studies on the molecular mechanisms of inflammation, and the development of new anti-inflammatory agents, are required.
\end{abstract}

Keywords: Cancer cachexia, immunosuppression, SIRS, CARS, MARS

(Received March 26, 2018; Accepted April 19, 2018)

\section{Introduction}

Cachexia is a multifactorial and multi-organ syndrome characterized by continuous, involuntary weight loss and systemic inflammation ${ }^{1,2)}$. These multiple symptoms were first described about 2000 years ago by Hippocrates, and are now a common feature of several diseases, such as chronic obstructive pulmonary disease, chronic heart failure, chronic infection, chronic renal failure, AIDS and cancer $^{3}$. Cachexia appears in approximately $50 \%$ of all cancer patients, who have been reported to experience reduced tolerance to treatment, therapeutic responses, quality of life and survival. The definition of cachexia has only recently been proposed. In 2011, an international consensus defined cancer cachexia (EPCRC: European Palliative Care Research Collaborative), which is commonly seen in clinics, as a multifactorial syndrome characterized by ongoing loss of skeletal muscle mass that

Corresponding author: Masahiko Shibata, MD, PhD. Department of Advanced Cancer Immunotherapy, Fukushima Medical University. 1 Hikarigaoka, Fukushima City, Fukushima 960-1295, Japan Phone: +81-24-547-1260, FAX: +81-24-5485643, E-mail: mshibata@fmu.ac.jp cannot be fully reversed by conventional nutritional support, and leads to progressive functional impairment ${ }^{1,4,5)}$. In the EPCRC study, cachectic patients had significantly higher inflammation, lower nutritional intake and performance status and shorter survival. The patients were also classified into 4 groups, no cachexia, pre-cachexia, cachexia and refractory cachexia and it was reported that the pre-cachexia stage might be better defined by additional factors representing the cachexia domain, for instance CRP and appetite loss. The authors concluded that a clear definition of pre-cachexia is needed, especially because this group is the target of intervention trials. The consensus guidelines were quickly applied to and validated in clinical practice. Furthermore, additional assessments and management algorithms were proposed ${ }^{6,7)}$. A diagnosis of cachexia can be made based on involuntary weight loss of $5 \%$ or more or, alternatively, a body mass index (BMI) of less than 20, accompanied by at least three of the following co-factors: decreased muscle strength, fatigue, anorexia, low fat-free mass, and abnormal biochemistry ${ }^{8,9)}$. However, the exact mechanisms of this syndrome remain unknown. Chronic inflammation appears to play a key role in inducing these changes and 
in disrupting appetite modulation. Additionally, several mediators that are either host- or tumor-derived, such as pro-inflammatory cytokines, eicosanoids, and hormones, have been implicated in the pathogenesis of cancer cachexia $^{10,11)}$.

An important feature of cachexia is chronic systemic inflammation and, paradoxically, immunosuppression ${ }^{12)}$. The compensatory humoral system that typically reduces excessive production of pro-inflammatory cytokines in order to maintain homeostasis is also highly activated in patients with cancer cachexia. After tissue injury, an initial systemic inflammatory response, termed systemic inflammatory response syndrome (SIRS), which is driven by high production of pro-inflammatory cytokines, appears. Subsequently, a compensatory system termed compensatory anti-inflammatory response syndrome (CARS), which is driven by anti-inflammatory cytokines and antagonistic proteins that are produced as a response, is activated ${ }^{13,14)}$. The above observations, along with cachexia, suggest that conditions similar to SIRS and CARS, or to mixed anti-inflammatory response syndrome (MARS), may exist in cases of cachexia. In the present review, these immunological mechanisms, and the resultant status of patients with cancer cachexia, are reviewed, and possible effective strategies using immune modulators against cachexia are discussed.

\section{Weight loss and metabolic changes}

Cancer cachexia is characterized by negative protein balance and the involuntary loss of lean body mass as well as systemic inflammation, and is clinically represented by significant weight loss. While the loss of skeletal muscle is the most obvious symptom of cancer cachexia, cardiac muscle is also depleted, and visceral muscle tends to be preserved. As we have mentioned above, systemic inflammation is one proposed major mechanism of cancer cachexia; there is an increase in pro-inflammatory cytokine levels in cancer cachexia.

The role of tumor necrosis factor (TNF)-alpha in mediating cancer cachexia is supported by evidence that intraperitoneal injection of a soluble recombinant human TNF-receptor antagonist improved food intake and weight gain in tumor-bearing rats ${ }^{15)}$. TNF-alpha increases gluconeogenesis, lipolysis and proteolysis, decreases the synthesis of proteins, lipids and glycogen, induces the formation of interleukin (IL)- ${ }^{16)}$, and stimulates the expression of uncoupling protein (UCP) 2 and UCP3 in cachectic skeletal muscles $^{17)}$.

IL-1 levels increase in the cachectic state and have been known to cause similar effects to those of TNFalpha ${ }^{18)}$. IL-6 is an important mediator in the defense mechanism of humans through its regulation of immune responses ${ }^{19)}$. Levels of IL-6 were observed to be higher in patients with cachexia than in weight-stable patients.
Although TNF-alpha or IL-6 may play important roles in the formation of cachexia, they are not considered to be solely responsive, and it is likely that a complex interplay of these factors is responsible for cachexia ${ }^{20,21)}$.

The metabolic changes found in cachexia resemble those of infection rather than starvation. Although weight loss caused by starvation is mainly from adipose tissue stores, weight loss in cancer cachexia patients is caused by the loss of body skeletal muscle and adipose tissue mass ${ }^{22}$. It has been widely observed that the rate of muscle protein catabolism increases in cachexia, while anabolism of new proteins decreases, resulting in net protein breakdown ${ }^{23,24)}$.

\section{Inflammatory and anti-inflammatory responses}

It has long been reported that the origin of cancer cachexia is rooted in systemic inflammation, not solely in the reduction of nutritional intake ${ }^{10,25)}$. We have reported that there are conditions of high production of pro-inflammatory cytokines such as IL-6 in patients with cancer cachexia compared with non-cachectic patients with cancer. In these cachectic patients, the production of the anti-inflammatory cytokine IL-10 is markedly increased. In addition, the serum level of proteins that act as antagonists of pro-inflammatory cytokines, including soluble receptor for tumor necrosis factor and IL-1 receptor antagonist, are increased in patients with gastric and colorectal cancers, along with an advancement of the diseases. The serum levels of these proteins were significantly inversely correlated to those of rapid turnover proteins (RTP) such as transferrin, prealbumin, and retinol binding protein, and were also correlated to the suppression of cell-mediated immune response and poor survival $^{26-29)}$. We have previously reported that the serum concentration of soluble receptors for IL-2 (sIL-2R) is also significantly increased and inversely correlated to RTP in patients with cancer cachexia ${ }^{27,30)}$. sIL-2R was proven to act as an antagonist of IL-2, and thus inhibits IL-2 action in the initiation, maintenance, and strengthening of cell-mediated immune function. Moreover, galectin-3, which has been implicated in cell growth, differentiation, apoptosis, adhesion, malignant transformation and angiogenesis, is increased in pancreatobiliary cancer, and was associated with malnutrition, inflammation and poor prognosis in our previous study ${ }^{31)}$. Considering SIRS, CARS and MARS, the mechanisms of immunosuppression seen in patients with cancer cachexia might thus be at least partially induced by these pro- and antiinflammatory immune responses (SIRS, CARS, MARS and sIL-2R).

A review by Argiles and Lopez-Soriano ${ }^{32)}$ separated cytokines according to their function as either procachectic factors or anticachectic factors in order to further define their roles. The procachectic factors include IL-6, 
Interferon (IFN)-gamma, and TNF-alpha, which act by promoting tissue wasting, and the anticachectic factors include IL-4, IL-10, IFN-alpha, IL-12, IL-15 and insulinlike growth factor-alpha, which act in opposition to the procachectic factors in an attempt to stabilize this tissue breakdown. The anticachectic cytokines have been shown to ameliorate the effects of the procachectic factors to varying degrees. Therefore, a balance of these two types of factors may exist, and achievement of such a balance may be a target of clinical strategies.

\section{Immune alterations}

\section{a. Th2 Polarization}

Immune responses have been classified into two types: type I, which provides cell-mediated immune responses; and type II, which supports B helper functions and humoral immune responses. These responses are regulated by the Th1 and Th2 subsets of the CD4+T helper (Th) cells, respectively. The typical immunological status of patients with advanced stages of cancer has been demonstrated to be suppressed cell-mediated immunity, and a Th2-dominant condition may be one of the major causes of this suppression ${ }^{29,33,34)}$.

\section{b. MDSC}

Much progress and significant therapeutic advances have been made in the field of anti-cancer treatment in recent decades. Cancer immunotherapy entered a new era with the introduction of immune checkpoint inhibitors $^{35,36)}$. The discovery of programmed death-1 (PD-1) and the ligand-1 (PD-L1) has opened a door to modern cancer immunotherapy, and blocking of the PD-1 or PDL1 has led to a fast and fundamental paradigm shift in the entire strategy of cancer treatment ${ }^{37)}$. However, these remarkable outcomes are not yet achievable in all kinds of malignant diseases, and in these types of tumor, we need to overcome the immunosuppressing microenvironment seen in patients without response to immune checkpoint inhibitors.

In general, a proinflammatory tumor microenvironment, as well as infiltrating CD8-expressing T lymphocytes, are associated with improved clinical outcomes in various types of tumor. In contrast, the inhibitory function of other immune cells such as myeloid-derived suppressor cells (MDSCs) and regulatory T cells (Tregs), appear to play major roles in the negative local control of antitumor immune reaction, and are therefore associated with a worse outcome ${ }^{38)}$.

MDSCs are a population of immature myeloid cells that are expanded in various conditions, including cancer and inflammation, and are capable of immunosuppres$\operatorname{sion}^{39-41)}$. Infiltrating MDSCs are now thought to be potent regulators of tumor-associated immunosuppression, tumor cell invasion, metastasis, and angiogenesis. The mechanisms of immunosuppression by MDSCs include the production of arginase 1, reactive oxygen species, and nitric oxide, as well as the secretion of immunosuppressive cytokines such as IL-10. MDSCs have been reported to grow at dramatically high rates within tumors, producing excessive quantities of inflammatory cytokines ${ }^{35,36)}$. Such an increase in MDSCs has been reported to be associated with cachexia ${ }^{37,38)}$. These cells and other cells of the innate immune system respond to tumors by producing TNF-alpha, IL-1 beta, IL-6 and IFN-gamma in an effort to stimulate the host's immune response and overcome pathogens ${ }^{39)}$. We recently reported that circulating levels of MDSCs were significantly increased in patients with gastrointestinal, breast, pulmonary, ovarian and thyroid cancers. In particular, MDSCs levels were increased in patients with cachexia, and were significantly correlated with immunosuppression and nutrition derangements such as decreases in RTP and inflammation ${ }^{40-43)}$. In thyroid cancer, circulating levels of MDSCs were significantly higher in patients with anaplastic carcinoma, and both of CRP and the production of IL-10 were significantly higher and albumin levels were lower in patients with higher MDSCs levels ${ }^{41)}$. Moreover, we have found that systemic inflammation underlying the accumulation of MDSCs is, at least partially, driven by IL-17, proinflammatory cytokine which is initially reported to be origin of the inflammation associating with autoimmune diseases, and vascular endothelial growth factor (VEGF). MDSCs, IL-17 and VEGF have shown to be significantly correlated with each other and these three inflammationrelating factors importantly showed to be prognostic indicators $^{44-47)}$.

\section{Strategies against cancer cachexia}

\section{a. Nutritional and metabolic support}

Nutritional interventions should be an essential part of the multimodal approach to cancer cachexia. Reduction in food intake is an important factor in developing cancer cachexia and weight loss, and muscle wasting may be reversible when nutritional and metabolic support are started at an early stage of cancer cachexia, rather than being delayed until an advanced stage of weight loss. All patients are recommended to have their nutrition monitored by both an oncologist and a clinical nutrition unit as soon as a diagnosis of cancer is made. Nutritional and metabolic support may be started with nutritional counseling, administration of oral supplements, nutraceuticals and artificial nutrition after monitoring nutritional state.

Cancer cachexia is different from simple starvation. Both inflammation and metabolic abnormality may alter the anabolic response of the skeletal muscles after meal ingestion. Recently, it has been reported that there is a strong rationale for early nutritional intervention for patients who have anabolic potential. No anabolic 
resistance has been reported to exist in cachectic cancer patients, and for such patients the high anabolic potential of dietary essential amino acids or specially formulated nutritional supplements containing high protein and high leucine are effective in preventing muscle loss ${ }^{48-52)}$. Van Dijk reported that cachectic patients with pancreatic cancer have a higher basal protein turnover, and that the different pattern of protein kinetics is primarily related to protein breakdown ${ }^{53)}$.

\section{b. Anti-inflammatory treatments against cancer ca- chexia}

There are traditional or established agents for cachexia treatment, such as megestrol acetate, medroxyprogesterone, ghrelin, cannabinoids, corticosteroids or dietary treatments that include omega-3-fatty acids and eicosapentaenoic acid. Other treatments especially proven to be related to reduction of inflammation are focused on in this review. As mentioned before, TNF-alpha, IL-6 and IFN-gamma have been implicated in the pathogenesis of cachexia, as well as in murine models, and antibody treatments for these cytokines can attenuate the disease's progress, although they cannot stop or reverse cancer cachexia ${ }^{54-56)}$.

Thalidomide (a-N-phthalimidoglutaramide) has complex immune-modulatory and anti-inflammatory actions. It has been shown to downregulate the production of TNF-alpha and other pro-inflammatory cytokines in monocytes, to inhibit the transcription factor nuclear factor kappa B (NFxB), to downregulate cyclooxygenase 2, and to inhibit angiogenesis ${ }^{57,58)}$.

Etanercept, a soluble p75 tumor necrosis factor receptor: FC (TNF-alpha decoy receptor) fusion protein for plasma cytokines, has been used over the last decade to treat immune-related symptoms of rheumatoid arthritis. Monk reported that patients with several advanced malignancies treated with etanercept combined with docetaxel had less fatigue and improved tolerability to chemotherapy $^{59)}$.

Several studies have investigated the effects of nonsteroidal anti-inflammatory drugs (NSAIDs) on the clinical findings of cancer patients. These studies reported improved quality of life, performance status, inflammatory markers, weight gain and survival ${ }^{60-62)}$. However, it was also reported that the concluded data were insufficient for recommending the widespread use of NSAIDs in practice ${ }^{63,64)}$.

\section{Conclusion and future directions}

No effective therapy against cancer cachexia is available at present. For this reason, it is mandatory to implement strategies aimed at preventing, or at least delaying, this condition. In this regard, the increasing knowledge about the molecular mechanisms of systemic inflamma- tion underlying cancer cachexia has allowed the identification of several potentially therapeutic targets, as well as the development of many promising drugs.

\section{References}

1) Fearon K, Strasser F, Anker SD, Bosaeus I, Bruera E, Fainsinger RI, Jatoi A, Loprinzi C, MacDonald N, Mantovani G, Davis M, Muscaritoli M, Ottery F, Radbruch L, Ravasco P, Walsh D, Wilcock A, Kaasa S, Baracos VE. (2011) Definition and classification of cancer cachexia: an international consensus. Lancet Oncol 12: 489495.

2) Argiles JM, Busquets S, Stemmler B, Lopes-Soriano FJ. (2015) Cachexia and sarcopenia: mechanisms and potential targets for intervention. Curr Opin Pharmacol 22: 100-106.

3) Fearon K, Arends J, Baracos V. (2013) Understanding the mechanisms and treatment options in cancer cachexia. Nat Rev Clin Oncol 10: 90-99.

4) Blum D, Stene GB, Solheim TS, Fayers P, Hjermstad MJ, Baracos VE, Fearon K, Strasser F, Kaasa S, on behalf of Euro-Impact (2014) Validation of the consensus-definition for cancer cachexia and evaluation of a classification model-a study based on data from an international multicenter project (EPCRC-CSA). Ann Oncol 25: 1635-1642.

5) Kaasa S, Loge JH, Fayers P, Caraceni A, Strasser F, Hjermstad MJ, Higginson I, Radbruch L, Haugen DF. (2008) Symptom assessment in palliative care: A need for international collaboration. J Clin Oncol 26: 3867-3873.

6) Vigano A, Del Fabbro E, Bruera E, Borod M. (2012) The cachexia clinic: from staging to menaging nutritional and functional problems in advanced cancer patients. Crit Rev Oncog 17: 293-303.

7) Blum D, Stene GB, Solheim TS, Fayers P, Hjermstad MJ, Baracos VE, Fearon K, Strasser F, Kaasa S, Euro I. (2014) Validation of the consensus-definition for cancer cachexia and evaluation of a classification model- a study based on data from an international multicentre project (EPCRC-CSA). Ann Oncol 25: 1635-1642.

8) Coussens LM, Werb Z. (2002) Inflammation and cancer. Nature 420: 860-867.

9) Baracos VE. (2011) Pitfalls in defining and qualifying cachexia. J Cachexia Sarcopenia Muscle 2: 71-73.

10) Evans WJ, Morley JE, Argiles J, Bales C, Baracos V, Guttridge D, Jatoi A, Kalantar-Zadeh K, Lochs H, Mantovani G, Marks D, Mitch WE, Muscaritoli M, Najand A, Ponikowski P, Rossi Fanelli F, Schambelan M, Schols A, Schuster M, Thomas D, Wolfe R, Anker SD. (2008) Cachexia, a new definition. Clin Nutr 27: 793-799.

11) Moldawer LL, Copeland EM III. (1997) Proinflammatory cytokines, nutritional support, and the cachexia syndrome. Interactions and therapeutic options. Cancer 79: 1828-1839.

12) Faber J, Uitdehaag MJ, Spaander M, Van Steenbergen-Langeveld S, Vos P, Berghout M, Lamers C, Rumke H, Tilanus H, Siersema P, Van Helvoort A, Van der Gaast A. (2015) Improved body weight and performance status and reduced serum PGE2 levels after nutritional intervention with a specific medical food in newly diagnosed patients with esophageal cancer or adenocarcinoma of the gastroesophageal junction. J Cachexia Sarcopenia Muscle 6: 32-44.

13) Bone RC, Grodzin CJ, Balk RA. (1997) A new hypothesis for pathogenesis of the disease process. Chest 112: 235-243.

14) Bone RC. (1996) Sir Isaac Newton, sepsis, SIRS, and CARS. Crit Care Med 24: 1125-1128.

15) Torelli GF, Meguid MM, Moldawer LL, Edwards CK, Kim HJ, Carter JL, Laviano A, Rossi Fanelli F. (1999) Use of recombinant human soluble TNF receptor in anorectic tumor-bearing rats. Am J Physiol 277: R850-R855.

16) Tijerina AJ. (2004) The biochemical basis of metabolism in cancer cachexia. Dimens Crit Care Nurs 23: 237-243.

17) Giordano A, Calvani M, Petillo O, Carteni M, Melone MR, Peluso G. (2003) Skeletal muscle metabolism in physiology and in cancer disease. J Cell Biochem 90: 170-186. 
18) Yeh SS, Schuster MW. (1999) Geriatric cachexia: the role of cytokines. Am J Clin Nutr 70: 183-197.

19) Mihara M, Hashizume M, Yoshida H, Suzuki M, Shiina M. (2012) IL-6/IL-6 receptor system and its role in physiological and pathological conditions. Clin Sci (London) 122: 143-159.

20) Tisdale MJ. (1997) Biology of cachexia. J Natl Cancer Inst 89: 1763-1773.

21) Barber MD, Fearon KC, Tisdale MJ, McMillan DC, Ross JA. (2001) Effect of a fish oil-enriched nutritional supplement on metabolic mediators in patients with pancreatic cancer cachexia. Nutr Cancer 40: $118-124$.

22) Dworzak F, Ferrari P, Gavacci C, Maiorana C, Bozzetti F. (1998) Effects of cachexia due to cancer on whole body and skeletal muscle protein turnover. Cancer 82: 42-48.

23) Tisdale MJ. (2001) Loss of skeletal muscle in cancer: biochemical mechanisms. Front Biosci 6: D164-D174.

24) Acharyya S, Ladner KJ, Nelson LL, Damrauer J, Reiser PJ, Swoap S, Guttridge DC. (2004) Cancer cachexia is regulated by selective targeting of skeletal muscle gene products. J Clin Invest 114: 370 378.

25) Thomas DR. (2007) Loss of skeletal muscle mass in aging: examining the relationship of starvation, sarcopenia, and cachexia. Clin Nutr 26: 389-399.

26) Shibata M, Takekawa M, Amano S. (1998) Increased serum concentrations of soluble tumor necrosis factor receptor I in noncachectic and cachectic patients with advanced gastric and colorectal cancer. Surg Today 28: 884-888.

27) Shibata M and Takekawa M. (1999) Increased serum concentration of circulating soluble receptor for interleukin-2 and its effect as a prognostic indicator in cachectic patients with gastric and colorectal cancer. Oncology 56: 54-58.

28) Shibata M, Nagata Y, Kimura T, Kanou H, Nezu T, Takekawa M, Fukuzawa M. (2000) Elevated serum concentration of interleukin-1 receptor antagonist (IL-1ra) is correlated to interleukin-6 and to hypoalbuminemia in cachectic patients with colorectal cancer. Int J Clin Oncol 5: 116-120.

29) Shibata M, Nezu T, Kanou H, Abe H, Takekawa M, Fukuzawa M. (2002) Decreased production of interleukin-12 and type 2 immune responses are marked in cachectic patients with colorectal and gastric cancer. J Clin Gastroenterol 34: 416-420.

30) Gonda K, Shibata M, Shimura T, Machida T, Suzuki S, Nakamura I, Ohki S, Sakurai K, Ohto H, Tomita R, Takenoshira S. (2012) Serum soluble interleukin-2 receptor is increased in malnourished and immunosuppressed patients with gastric and colorectal cancer: possible influence of myeloid-derived suppressor cells. World J Oncol 3: 158-164.

31) Shimura T, Shibata M, Gonda K, Kofunato Y, Okada R, Ishigame T, Kimura T, Kenjo A, Kono K, Marubashi S. Significance of circulating galectin-3 in pancreatobiliary cancer. (2017) Anticancer Res 37: 4979-4986.

32) Argiles JM, Lopez-Soriano FJ. (1999) The role of cytokines in cancer cachexia. Med Res Rev19: 223-248.

33) Shibata M, Nezu T, Takekawa M, Takizawa H, Ando K, Miyake H, Amano S, Kurosu Y. (1996) Serum levels of interleukin-10 and interleukin-12 in patients with colorectal cancer. Annals N Y Acad Sci 795: 410-412.

34) Lakshmi Narendra B, Eshvendar Reddy K, Shantikumar S, Ramakrishna S. (2013) Immune system: a double-edged sword in cancer. Inflamm Res 62: 823-834.

35) Couzin-Frankel J. (2013) Breakthrough of the year 2013. Cancer immunotherapy Science 342: 1432-1433.

36) Mahoney KM, Freeman GJ, McDermott DF. (2015) The next immune checkpoint inhibitors: PD-1/PD-L1 blockade in melanoma. Clin Ther 37: 764-782.

37) Pennock GK, Chow LQ. (2015) The evolving role of immune checkpoint inhibitors in cancer treatment. Oncologist 20: 812-822.

38) Gajewski TF, Schreiber H, Fu Y-X. (2013) Innate and adaptive immune cells in the tumor microenvironment. Nat Immunol 14: 10141022 .

39) Kamo I, Friedman H. (1977) Immunosuppression and the role of suppressive factors in cancer. Adv Cancer Res 25: 271-321.

40) Ohki S, Shibata M, Gonda K, Machida T, Shimura T, Nakamura I, Ohtake T, Koyama Y, Suzuki S, Ohto H, Takenoshita S. (2012) Circulating myeloid-derived suppressor cells are increased and correlate to immune suppression, inflammation and hypoproteinemia in patients with cancer. Oncol Rep 28: 453-458.

41) Suzuki S, Shibata M, Gonda K, Kanke Y, Ashizawa M, Ujiie D, Suzushino S, Nakano K, Fukushima T, Sakurai K, Tomita R, Kumamoto K, Takenoshita S. (2013) Immunosuppression involving increased myeloid-derived suppressor cell levels, systemic inflammation and hypoalbuminemia are present in patients with anaplastic thyroid cancer. Mol Clin Oncol 1: 959-964.

42) Yazawa T, Shibata M, Gonda K, Machida T, Suzuki S, Kenjo A, Nakamura I, Tsuchiya T, Koyama Y, Sakurai K, Tomita R, Ohto H, Gotoh M, Takenoshita S. (2013) Increased IL-17 production correlates with both immunosuppression involving MDSC and nutritional impairment in patients with various gastrointestinal cancers. Mol Clin Oncol 1: 675-679.

43) Nakamura I, Shibata M, Gonda K, Yazawa T, Shimura T, Anazawa T, Suzuki S, Sakurai K, Koyama Y, Ohto H, Tomita R, Gotoh M, Takenoshita S. (2013) Serum levels of vascular endothelial growth factor are increased and correlate with malnutrition, immunosuppression, involving MDSCs and inflammation in patients with cancer of the digestive system. Oncol Lett 5: 1682-1686.

44) Watanabe T, Shibata M, Nishiyama H, Soeda S, Furukawa S, Gonda K, Takenoshita S, Fujimori K. (2014) Serum levels of rapid turnover protein are decreased and related to systemic inflammation in patients with ovarian cancer. Oncol Lett 7: 373-377.

45) Gonda K, Shibata M, Ohtake T, Matsumoto Y, Tachibana K, Abe N, Ohto H, Sakurai K, Takenoshita S. (2017) Myeloid-derived suppressor cells are increased and correlated with type 2 immune responses, malnutrition, inflammation, and poor prognosis in patients with breast cancer. Oncol Lett 14: 1766-1774.

46) Minamikawa K, Shibata M, Gonda K, Ujiie D, Ashizawa M, Nakajima T, Okayama H, Sakamoto W, Saito M, Momma T, Mimura K, Ohki S, Shimura T, Ohto H, Takenoshita S, Kono K. (2017) IL-17 and VEGF are significantly associated with disease progression involving systemic inflammation in patients with gastric and colorectal cancers. Ann Cancer Res Ther 25: 67-76.

47) Tachibana K, Shibata M, Gonda K, Matsumoto $Y$, Nakajima T, Ohtake T, Ohto H, Kono K, Takenoshita S. (2017) IL-17 and VEGF are increased and correlated to systemic inflammation, immune suppression, and malnutrition in patients with breast cancer. Eur J Inflammation 20: 1-10.

48) Engelen MP, Safar AM, Bartter T, Koeman F, Deutz NE. (2015) High anabolic potential of essential amino acid mixtures in advanced nonsmall cell lung cancer. Ann Oncol 26: 1960-1966.

49) Deutz NE, Safar A, Schutzler S, Memelink R, Ferrando A, Spencer H, van Helvoort A, Wolfe RR. (2011) Muscle protein synthesis in cancer patients can be stimulated with a specially formulated medical food. Clin Nutr 30: 759-768.

50) Chevalier S, Winter A. (2014) Do patients with advanced cancer have any potential for protein anabolism in response to amino acid therapy? Curr Opin Clin Nutr Metab Care 17: 213-218.

51) Engelen MP, van der Meij BS, Deutz NE. (2016) Protein anabolic resistance in cancer: does it really exist? Curr Opin Clin Nutr Metab Care 19: 39-47.

52) Aoyagi T, Terracina KP, Raza A, Matsubara H, Takabe K. (2015) Cancer cachexia, mechanism and treatment. World J Gastrointest Oncol 7: 17-29.

53) van Dijk DP, van de Poll MC, Moses AG, Preston T, Olde Damink SW, Rensen SS, Deutz NE, Soeters PB, Ross JA, Fearon KC, Dejong CH. (2015) Effects of oral meal feeding on whole body protein breakdown and protein synthesis in cachectic pancreatic cancer patients. J Cachexia Sarcopenia Muscle 6: 212-221.

54) Strassmann G, Fong M, Kenney JS, Jacob CO. (1992) Evidence for the involvement of interleukin 6 in experimental cancer cachexia. J Clin Invest 89: 1681-1684.

55) Matthys P, Heremans H, Opdenakker G, Billiau A. (1991) Antiinterferon-gamma antibody treatment, growth of Lewis lung tu- 
mours in mice and tumour-associated cachexia. Eur J Cancer 27: $182-187$.

56) Strassmann G, Kambayashi T. (1995) Inhibition of experimental cancer cachexia by anti-cytokine and anti-cytokine-receptor therapy. Cytokines Mol Ther 1: 107-113.

57) Sampaio EP, Sarno EN, Galilly R, Cohn ZA, Kaplan G. (1991) Thalidomide selectively inhibits tumor necrosis factor alpha production by stimulated human monocytes. J Exp Med 173: 699-703.

58) Gordon JN, Goggin PM. (2003) Thalidomide and its derivatives: emerging from the wilderness. Postgrad Med J 79: 127-132.

59) Monk JP, Phillips G, Waite R, Kuhn J, Schaaf LJ, Otterson GA, Guttridge D, Rhoades C, Shah M, Criswell T, Caligiuri MA, Villalona-Calero MA. (2006) Assessment of tumor necrosis factor alpha blockade as an intervention to improve tolerability of doseintensive chemotherapy in cancer patients. J Clin Oncol 24: 18521859.

60) Lai V, George J, Richey L, Kim HJ, Cannon T, Shores C, Couch M. (2008) Results of a pilot study of the effects of celecoxib on cancer cachexia patients in patients with cancer of the head, neck and gastrointestinal tract. Head Neck 30: 67-74.
61) McMillan DC, Wigmore SJ, Fearon KC, O'Gorman P, Wright CE, McArdle CS. (1999) A prospective randomized study of megestrol acetate and ibuprofen in gastrointestinal cancer patients with weight loss. Br J Cancer 79: 495-500.

62) Cerchietti LC, Navigante AH, Castro MA. (2007) Effects of eicosapentaenoic and docosahexaenoic n-3 fatty acids from fish oil and preferential Cox-2 inhibition on systemic syndromes in patients with advanced lung cancer. Nutr Cancer 59: 14-20.

63) Reid J, Hughes CM, Murray LJ, Parsons C Cantwell MM. (2013) Non-steroidal anti-inflammatory drugs for the treatment of cancer cachexia, a systematic review. Palliat Med 27: 295-303.

64) Solheim TS, Fearon KC, Blum D, Kaasa S. (2013) Non-steroidal anti-inflammatory treatment in cancer cachexia: a systematic literature review. Acta Oncol 52: 6-17. 